Current Research in Dentistry 1 (2): 19-22, 2010

ISSN 1949-0119

(C) 2010 Science Publications

\title{
Prevalence Study of Etiologies of Developmental Defects of Enamel of First Permanent Molar among Six to Seven Years Old Children
}

\author{
F. Farrokh Gisoo and A. Mohseni \\ Kerman Faculty Shafa Avnue Gomhouri, BLV Kerman, Iran
}

\begin{abstract}
Problem statement: The final product of amelogensis which generates in three subsequent phases is dental enamel. These Phases are divided into: excretory, calcification and maturation stage of enamel. While these specific complex events, are affected by specific agents, the act of amelogenic cells will be disturbed and hence the results of this process is improper and imperfect enamel production which vary in an spectrum from a little but discoloration to complete loss of enamel. Approach: The aim of this study was to determine the prevalence assessment of enamel defect in permanent first molar in 6-7 years student of Kerman city. Permanent first molar of students 844 boys and 793 girls was examined. Their demographic information and teeth information was collected in a questionnaire and questions to investigate etiology of hypoplasia in three stages were asked from the parents. Results: From 1637 students that enrolled in this study, 123 cases had enamel defect at least in one plan of one tooth. Conclusion: Prevalence of hypoplasia in two genders was approximately the same and there was no definitive statistical difference between them. Buccal place was the most affected plan and permanent first molar was the most affected tooth. Assessment of etiologies of hypoplasia, ten major factors was detected: 38.2, 26, 21.9, 13.8, 9.8 and $6.8 \%$ had history of cesarean section, childhood disease, hepatitis A infection in infantile period and administration of medicines by parent meanwhile pregnancy, maternal disease during pregnancy and immature birth, respectively.
\end{abstract}

Key words: Organic materials, systemic factors, kerman city, cesarean section, maternal disease, cesarean section, hypo plastic, enamel hypocalcification

\section{INTRODUCTION}

Final product of Amylogensis that performed in three sequential phases is dental enamel. It consists excretory, calcification and mature phases. In these phases, enamel that is composed of inorganic matrix converted to the network of organic crystals, such that, finally it consists $96 \%$ organic materials at the end.

When these cellular, physic-chemical events are affected by some special factors, the action of enamelproducing cells temporary or permanently turns pathologic and the result of this process is an enamel tissue that in quantity and quality is impaired.

Local or systemic factors interfere within making healthy matrix, they cause superficial enamel impairments and leading to hypoplasia abnormal states. The factors that interfere in calcification and enamel maturation, they caused the situation called enamel hypocalcification. The reason that many patients referred to dentist, is the color changing of tooth in anterior teeth. Because of affection of posterior teeth, most patients don,t pay attention to their color changing, so the dentist should warn them these teeth are ready to decay.

At the first phase, when the structure of teeth enamel is ruined, it is destroyed sooner. Sometimes, the progressing of quick decay and erosion is added to the main form. Secondly, the appearance of these teeth is not well and cosmetic; they are abnormal in form, color, appearance and size. Third, the teeth with notgood enamel are painful, sensitive and patient lead to malnutrition. Forth, the curve of external surface of teeth crown is not normal; therefore, there is continuous inappropriate, abnormal pressure on gum and periodontal tissues in different grades. Fifth, growth of hypo plastic teeth. The last problem is that the failure in enamel-structure cause the failure in lateral and anterior connection with adjacent and opposite teeth. There are different reasons for this failure; while, dentist might prevent many of them. Aim of current study was to endeavor to prevalence of enamel hypoplasia in the first permanent molar teeth in 6-7 year old students in Kerman and to evaluate the reasons of enamel hypoplasia in this group. 
Enamel hypoplasia is enamel defects that occur in one, some or all the teeth. Many researchers investigated the enamel defects, including enamel hypoplasia. Many factors are stated as etiologic in enamel hypoplasia. The study about DDE indices dates to one century, till now there are different studies on these indices related factors. In 1916, Mckay used the spotted enamel expression to explain the children teeth that lived in Colorado Springs. In 1931, fluoride amount of water supplies was measured by Chare child (1931), it resembled it as a etiologic factors. Dean (1935) corrected the previous classification of evolution failure (that consisted seven groups; from normal to severe) with omitting the "moderately severe" states (Sampson et al., 1992).

In 1942, he created the method for measuring the intense of spotted enamel; this means they measured every grade of spotted enamel with one statistical scale. In 1942, he examined the geographical distribution, in respect of fluoride in drinking water, the result determined in $1.2 \mathrm{ppm}$ fluoride rate, $33 \%$ of children had the slight grade of spotted enamel, meanwhile in 5 ppm fluoride or more than hundred percent of children had this disorder, also the intensity of involvement was worse (Sampson et al., 1992).

$\mathrm{Li}$ and Colleague (1989) in China examined 1344 teenager (13-15 year old). The researchers reported DDE index $(22.2 \%)$, they stated the central maxillar teeth $(40.8 \%)$ as the most prevalent involved teeth. They reported the canine of upper jaw (18.5\%), the first molar of down haw $(25.7 \%)$ as the prevalent teeth with abnormal- enamel. They realized that most of enamel failure is occurred in buccal surface than others (the prevalence of enamel opacity was 8.6\%) (Li et al., 1995).

Nazaka et al., (1990) examined persons of lower than twenty years old with enamel hypoplasia. They used the questionnaire and 111 persons in control group completed this questionnaire. To determine hypoplasia, they used DDE index, according to reported data, the number of hypoplastic teeth consisted one to 28 teeth. The most prevalent hypo-plastic teeth were upper central incisor. Because local factors were less presented in this survey, enamel hypoplasia in the most teeth was symmetrical (Nozaka et al., 1990).

In another research, Love and colleagues was done about the spreading of hypoplasia (1999) in 88 children (810 year old) with DDE indication in old kingdom, Egypt, they reported that $48 \%$ of persons had enamel hypoplasia, it consisted $17 \%$ of permanent teeth and $8 \%$ of milk teeth. The permanent teeth consisted $87 \%$ of entire counted permanent teeth. The enamel failure in upper central teeth had the most prevalent (Pindborg, 1970).
Safari and colleagues performed the research (1378) on 8-9 year-old students (Esfahan). They examined 800 students, 159 of 800 persons had evolution failures in teeth enamel. In this research, they used DDE indication, this indication was $19.9 \%$. It was reported that the prevalence in males was more than females.

The permanent teeth is 2.2 more than deciduous teeth and the maxilla teeth is 2.3 more than mandible teeth were prone to evolutional failure in enamel. Although, the problem in buccal and lingual levels of deciduous teeth reported equal. In permanent teeth, the problem in buccal side was 5.3 times more than in lingual side.

The prevalence of enamel opacity, hypoplasia, badcolor differing from opacities and other failures or combination of them was 49.12, 47, 2 and $1.9 \%$, respectively. Also the prevalence of all failures in permanent teeth was more than deciduous teeth except in the missing enamel hypoplasia (it is more in deciduous teeth). The most prevalence of evolution failures was seen in central maxillar teeth (33.2\%), the least one was in the first mandible permanent Molar $(2.4 \%)$.

\section{MATERIALS AND METHODS}

sample size for current study was 1637 persons (844 boys, 793 girls), by statistic measuring and considering the level of confidence $95 \%$, error rate $0.3 \%$, based on pilot investigation, with obtained variance of $70 \%$ according to previous studies.

This survey is a descriptive-analytic study. It consists gathering and presenting the qualitative data in order to show the prevalence of evolution failure in region. It is obvious that the study is non-experimental, but cross-sectional.

The participants were elementary school students from 6-7 year-old. After observation of the school, s record book, we omitted the students who were not in this age range. As soon as clusters rates were calculated in each region, sixteen schools were selected from the inspected regions.

Method of examination: The examination with falciform catheter (no. 23), the disposable oral mirror and forceps was done. The infected catheters was cleaned and washed (by water) and stored in special closed-door basket containing Microten solution for re-disinfection. We used two closed baskets for placement of the sterile and un-sterile devices. It was done in a room equipped used one desk, several chairs for examination of visitors were hired. The survey was conducted in a separated room. For recording the enamel damages findings, we 
used a questionnaire that was approved by the research council of dentistry faculty Kerman.

Meanwhile examination, first, the demographic and mouth health information were recorded by examiner and he recorded the kind of damages and the location of them. Then, parents that their children had enamel failure, invited to school, to be asked to fill another questionnaire. it consists information about etiologic factors pre- and post-natal and until one-year old.

Limitation of the study:

- Some teachers were not cooperative, because examination time interfered with lessons time, so we limited the examination time to break time

- Some students were not cooperative because of worrisome and anxiety, it was explained for them and it made them more cooperated

\section{RESULTS}

Totally, 1637 students (6-7 year-old) from Kerman city, 793 girls and 844 boys were enrolled in the current study. Among these samples, 123 persons had (7.5\%) at least one tooth with one enamel defect, among them 55 $(44.7 \%)$ and $67(55.3 \%)$ were girls and boys. The prevalence rates are presented in Table 1.

According to table one, the prevalence rate of enamel hypoplasia in girls was 793 (of total examined persons) $(6.9 \%)$, while this rate in boys was 844 $(8.1 \%)$. The prevalence of enamel defects in girls and boys were almost the same and there was not any statistically significant difference. The most involved location (the location of enamel failures) was buccal and then occlusal, lingual and proximal.

Table 1: Frequency of enamel defects among genders

\begin{tabular}{llll}
\hline & With defect $=$ & Without defect $=$ & Total $=$ \\
& $\mathrm{N}(\%)$ & $\mathrm{N}(\%)$ & $\mathrm{N}(\%)$ \\
\hline Male & $68(8.1)$ & $776(91.9)$ & $884(100)$ \\
Female & $55(6.9)$ & $738(93.1)$ & $793(100)$ \\
Total & $123(7.5)$ & $1514(92.5)$ & $1637(100)$ \\
\hline
\end{tabular}

Table 2: Frequency of defects of enamel in various teeth

\begin{tabular}{llll}
\hline Involved side & UL6=N $(\%)$ & UR6= N (\%) & LR6 =N (\%) \\
\hline Buccal & $16(13)$ & $15(12.2)$ & $22(17.9)$ \\
Eclosal & $2(1.6)$ & $5(4.1)$ & $9(7.3)$ \\
Lingual & $10(8.1)$ & $9(7.3)$ & $1(0.8)$ \\
Bucca Proximal & & & $1(0.8)$ \\
Buccal and Eclusal & $10(8.1)$ & $9(7.3)$ & $16(13)$ \\
$\begin{array}{l}\text { Proximal eclusal } \\
\text { Buccal and Lingual }\end{array}$ & $1(0.8)$ & $3(2.4)$ & $1(0.8)$ \\
Eclusal lingual & $2(1.6)$ & $1(0.8)$ & $1(0.8)$ \\
Buccal Eclusal lingual & $9(7.1)$ & $4(3.3)$ & $2(1.6)$ \\
\hline
\end{tabular}

Observation of the first permanent Molar tooth, in the left of upper jaw, the first permanent Molar tooth in the right of upper jaw and the first permanent Molar tooth in the right of bottom jaw demonstrated 43.9, 42.3 and $61 \%$ participants to have enamel dysplasia, respectively. This data is presented in Table 2

According to these results, we might suggest that the most enamel defects was observed in the first permanent Molar and then in the right of bottom jaw. About the dysplasia prevalence, according to mother illness meanwhile the pregnancy period, it was observed twelve cases (9.8\%) with enamel failure, reported that mothers (in pregnancy period) had illnesses like; hypothyroidism, rubella, goiter, asthma.

Assessment of the relation between enamel dysplasia prevalence with consumed drugs of mother in pregnancy period, showed that the mothers of 17 participants with enamel failure, had history of medicines like; penicillin, ibuprofen and acetaminophen. The relation between enamel dysplasia and type of delivery, 76 children $(6.8 \%)$ with enamel failure were born in normal and 47 with caesarean section. The relation between hypoplasia prevalence and neonatal icterus, 27 persons $(21.9 \%)$ with enamel failure had neonatal icterus in infantile period, versus 96 cases $(78 \%)$ without it.

The relation between hypoplasia prevalence and neonatal illness, 32 children $(26 \%)$ with enamel failure reported disease like; diabetes, ear infection, hyper pyrexia and 91 persons $(74 \%)$ had not any of above disease. In evaluating the relation between enamel hypoplasia and consumed drugs until one year old, 27 person $(21.9 \%)$ had history of consumed the drugs like; antibiotics, salbutamol spray and nystatin.

Considering the prevalence of enamel hypoplasia within children with history of premature birth, eight cases $(6.5 \%)$ with enamel failure were born immature and $93.5 \%$ of cases with enamel failure had not this problem.

\section{DISCUSSION}

Enamel production occurs in two stages and local or systemic factors interfere in both enamel matrix and classification formation, respectively cause the enamel hypoplasia or hypoclacification.

If hypoplasia is caused by environmental factors, it might be seen in one or multiple teeth, but if it is caused by genetic factors, it,s seen in almost all teeth (deciduous and permanent (Sampson et al., 1992).

In this study, the prevalence of enamel hypoplasia in the first permanent Molar,s (in boys and girls) were 7.5\%. Li et al., (1995) examined 1344 juveniles (13-15 year-old), they reported DDE index as $22.2 \%$. In 
another research, Warankulas (1989) examined 79 juveniles (15-16 year-old) who lived in Glamorgan, the DDE index was reported as 10.2. In other research, Love and Colleagues examined 88 children (8-10 yearold) in old kingdom, Lovell and Whyte (1999) for hypoplasia prevalence, the DDE index was reported $17 \%$ in permanent teeth. The diversity of enamel hypoplasia prevalence in different cities and countries would be attributed to different factors that induce enamel failure, such as Floide of drinking water.

In this study, the prevalence of enamel failure was almost equal in boys and girls; there is not significant difference statistically. So, it seems gender doesn,t play role in the prevalence of enamel failure. Previous studies were done by other researchers (Sampson et al., 1992; Warnakulasuriya, 1989; Mortazavi et al., 1995).

The results of current study showed the prevalence of enamel defect in buccal level more than other sides, other studies done before, confirmed this (Sampson et al., 1992). Also in this study, the prevalence of enamel hypoplasia in the lower jaw was more than upper jaw. However, the previous studies showed the prevalence of enamel hypoplasia in the upper jaw, more than the lower (Sampson et al., 1992; Mortazavi et al., 1995). While, no reason explained in literature for this notion.

It was observed that $38.2 \%$ of participants with enamel defect were born with caesarean section. Afshar and colleagues studied the impact of delivery type on the occurrence of first permanent Molar defect, from the child birth files. They reported $26 \%$ of children who gave birth with caesarean, had enamel failure in the first Molar, while children who gave birth in normal delivery had $7 \%$ first Molar involvement. In this study, 26\% of participants with enamel defect, reported that during infantile period, until one year old have had diseases like; diabetes, hyperpyrexia, asthma and diarrhea,. Foroghi and colleagues, showed the prevalence of enamel defect in the first permanent Molar and infantile infection diseases, 23.3\% (Sampson et al., 1992)

In this STUDY, $9.8 \%$ of participants with enamel defect were those whom mothers had diabetes, hyperthyroidism, rubella or asthma twenty eight percent of diabetic mothers showed hypoplasia, while just nine percent of children whose gestational diabetes was controlled in mothers, showed enamel hypoplasia.

\section{CONCLUSION}

In this study, we provided the questionnaire participants with enamel defect, we didn,t have control group, so we just examined the reasons of dysplasia prevalence in ill children. Each of above items can cause the enamel defect. Regarding teeth are essential for appropriate occlusion, oral health should be cared lifelong. The enamel defect can be caused by environmental and systemic factors like, lake of nutrition, congenital rubella, premature baby or trauma and the pediatric dentist should warn parents about these factors, he should educate them about these risk factors in pregnancy and after birth to prevent them. Because these risks affecting the teeth, make them vulnerable to erosion and decay. So, the teeth with enamel dysplasia need better oral health to prevent early decaying.

\section{REFERENCES}

Li, Y., J.M. Navia and J.Y. Bian, 1995. Prevalence distribution developmental enamel defects in primary dentition of Chinese children 3-5 years old. Community Dent. Oral Epidemiol., 2: 72-79. PMID: 7781303

Lovell, N.C. and I. Whyte, 1999. Patterns of dental enamel defects at ancient Mendes, Egypt. Am. J. Phys. Anthropol. 1: 69-80. PMID: 10490469

Mortazavi, S.H., M.A. Beach, J.A. Jones and J.P. McGeehan, 1995. Bit error simulation of DQPSK for a slow frequency hopping CDMA system in mobile radio communications. Proceedings of the 6th IEEE International Symposium Personal, Indoor and Mobile Radio Communications, 'Wireless: Merging onto the Information Superhighway', Sep. 27-29, Toronto, Ont., Canada, pp: 183-187.

Nozaka, K., T. Sato and T. Mukaida, 1990. Clinical study of enamel hypoplasia and its causes. 1. Primary teeth. Shoni Shikagaku Zasshi, 3: 561-578. PMID: 2133959

Pindborg, J.J., 1970. Pathology of the dental Hard Tissues. 1st Edn., W. B. Saunders Company, Philadelphia, PA. USA., pp: 443. http://www.amazon.com/Pathology-Dental-HardTissues-

Pindborg/dp/B00138LVQU/ref=sr_1_3? ie=UTF8 \&qid=1309926717\&sr=8-3

Sampson, J.R., D. Attwood, A.S. Al Mughery and J.S. Reid, 1992. Pitted enamel hypoplasia in tuberous sclerosis. Clin. Genet., 42: 50-52. DOI: 10.1111/j.1399-0004.1992.tb03137.x

Warnakulasuriya, K.A., 1989. Prevalence of selected developmental dental anomalies in children, in Sri Lanka. ASDC J. Dent. Child., 2: 137-139. PMID: 2786012 